Journal of Case Reports 2018;8(4):240-243

\title{
Varied Neurological Presentations of Common Krait Bite in an Adolescent
}

\author{
Md Sohail Hassan ${ }^{1}$, Sisir Chakroborty ${ }^{2}$, Kaushik Ghosh ${ }^{3}$, Ambarish Bhattacharya ${ }^{4}$ \\ ${ }^{1}$ Department of Stroke Medicine, Worcester Royal Hospital, Worcestershire, WR5 1DD, United Kingdom; Department of Medicine, \\ ${ }^{2}$ College of Medicine and Sagore Dutta Hospital, ${ }^{3}$ Murshidabad Medical College, ${ }^{4}$ Charnok Hospital, West Bengal, India.
}

Corresponding Author:

Dr. Kaushik Ghosh

Email: drkaushikghosh@gmail.com

This is an Open Access article distributed under the terms of the Creative Commons Attribution License (creativecommons.org/ licenses/by/3.0).

Received

Accepted

Published

February 22, 2018

September 30, 2018

October 20, 2018

\begin{abstract}
Background: Snakebite can cause a galaxy of neurological symptoms sometimes in combination with hematotoxic features. Case Report: We report a 13 year old boy who was accidentally bitten by a common krait. Initial presentation showed evidence of neurotoxicity and paralysis which was managed as per current guidelines and the patient showed immediate response and clinical improvement only to develop delayed neurotoxicity and hematotoxicity with deterioration after initial response. However, the patient could be managed with available medical care and discharged in a stable condition. Conclusion: Such bizarre manifestations of neurotoxicity due to two different etiology in single krait bite is extremely rare and will add to the knowledge of existing literature.
\end{abstract}

Keywords: Adolescent, Cerebral Infarction, Snake Bites, Stroke, Paralysis.

\section{Introduction}

Snake bites are common occurrence throughout the world. Although some patients die due to severe envenomation, most patients die due to lack availability of point-of-care at their locations. The areas of the world having the highest rates of snakebites are amongst the most poorly developed and the staffs at these locations need to be properly trained regarding the management of this disorder. Most nations (including India) have their own guidelines regarding the management of snakebites which is accordance to the species that are prevalent in those locations and availability of anti-venom type. Common krait is one of the most commonly available poisonous snakes in India. It is important that all healthcare workers are aware of the fact that this type of snakebite can cause a galaxy of neurological symptoms sometimes in combination with hematotoxic features as well.

\section{Case Report}

A 13 years old male child from the Kalna, Burdwan district, West Bengal presented to the Emergency department of Burdwan Medical College with a history of Gokhura (common krait) snake bite in the left lower leg on the afternoon while returning from the playground. The boy did not have any pre-existing illness and was apparently well until this incident. The bite was unprovoked around a bush on the side ways.

Initially the patient had been taken to a rural health centre within 2 to 3 hours but by that time he had started developing drooping of both eyelids, inability to drink and swallow solid food as well as diplopia. However, the power of all four limbs was normal and deep tendon reflexes were preserved. No abnormality was detected in the higher mental function, sensory and autonomic examination. Mild swelling along with local erythema was present at the bite site. Initial management with anti-venom was started along with other supportive care and the patient sent to tertiary medical facility. Patient's vitals were stable till then.

On admission at Burdwan, the patient was drowsy, cyanosed and unable to communicate 
verbally. His pulse rate was 104/minute, blood pressure 90/68 $\mathrm{mm} \mathrm{Hg}$, respiratory rate was 28/ minute (shallow and irregular), $\mathrm{SpO}_{2} 64 \%$ (on room air). There were bilateral scattered crepitations on chest auscultation. On neurological examination pupils were normally reacting to light bilaterally but bilateral non-responsive planter reflex. Immediately patient was shifted to intensive care unit, intubated and put on mechanical ventilatory support (assist control mode). Inotropic support (nor-adrenaline) was also given to maintain hemodynamic stability. Pharmacotherapy with antibiotics, supportive care and anti-venom were started simultaneously. Over next 24 hours the patient's condition gradually improved and mechanical ventilatory support tapered. On next day patient recovered mostly from neuroparalytic symptoms, regained spontaneous respiratory drive and cough reflex. He could be extubated successfully after 48 hours of admission.

On third day of admission patient developed fever along with left sided facial deviation. Patient was hemodynamically stable with systolic blood pressure $104 \mathrm{~mm}$ of $\mathrm{Hg}$, heart rate $84 /$ minute, $\mathrm{SpO}_{2}$ 93\% (on room air). On neurological examination there was new onset upper motor neuron (UMN) type right $7^{\text {th }}$ cranial nerve palsy [Fig.1] without bulbar muscle involvement. Patient's higher mental function, motor, sensory examinations were absolutely normal. No other systemic abnormalities were found. From fifth day onwards the fever subsided but there was persistent facial deviation. Local swelling and erythema also gradually decreased over time and the patient discharged on ninth day of treatment in hemodynamically stable condition with residual UMN right facial nerve palsy which almost completely subsided at two weeks follow up.

Initial investigations like routine blood and urine examinations were sent along with whole blood clotting time. There was albuminuria and RBC 1-2/ HPF in urinalysis but the initial whole blood clotting time (WBCT) was less than 20 mins. Coagulation profile showed prothrombin

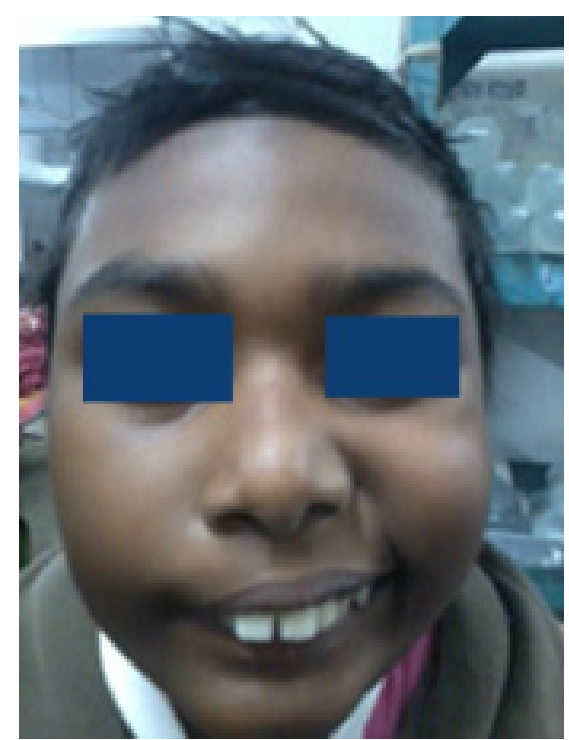

Fig.1: UMN type right $7^{\text {th }}$ cranial nerve palsy.

time (PT): 23 minutes 34 seconds, international normalized ratio (INR): 2.1, partial thromboplastin time (PTT): 39 minutes 40 seconds, bleeding time (BT): 4 minutes 30 seconds, clotting time (CT): 11 minutes 43 seconds. Thereafter, whole blood clotting test (WBCT) was performed every six hours till patient became asymptomatic. On third day when patient developed fever, to rule out hospital acquired infection and to localize source of infection, urine routine examination along with culture, blood for complete hemogram, malarial parasite dual antigen test, chest X-ray (PA view), swab for culture from site of bite were sent for examination but all reports came normal. Hence, MRI brain and CSF examination were ordered. CSF examination revealed mild elevation of protein (51 $\mathrm{mgm} / \mathrm{dL}$ ) with cell count 20 of which $80 \%$ were mononuclear and remaining 20\% were neutrophils and other parameters were within normal limits. MRI brain revealed that there was left middle cerebral artery (MCA) acute infract with right parietal petechial hemorrhage [Fig.2]. Bilateral carotid artery Doppler study, echocardiography to rule pre-existing cardiac disease, serum lipid profile, factor $\mathrm{V}$ level, homocysteine level in serum and urine, protein $\mathrm{C}$ and $\mathrm{S}$ level failed to establish any underlying pathology. Serum serological 
examinations for $\mathrm{HBsAg}$, anti-HCV, HIV were non-reactive.

Initial management was local wound toileting, tetanus toxoid and analgesics. He was treated with $100 \mathrm{ml}$ (10 vials) polyvalent antisnake venom slow infusion every six hours on the basis of neuroparalytic symptoms and whole blood clotting time results. He was also started on intravenous neostigmine $0.5 \mathrm{mg}$ with injection atropine $0.6 \mathrm{mg}$ at regular interval till the muscular weakness resolved.

The patient was treated initially in intensive care unit (ICU) where he was initially intubated and put on mechanical ventilatory support (assist control mode). Inotropic support with injection noradrenaline infusion along with intravenous fluid resuscitation was also given to maintain hemodynamic stability. Supportive care with intravenous ceftriaxone $1 \mathrm{~g}$ per day, intravenous ranitidine was also given. Physiotherapy and rehabilitation was started in the hospital and continued at home. Patient's condition stabilized and he was discharged with some residual left sided facial paralysis. On follow-up after two weeks he was clinically normal with just minor facial deviation.

\section{Discussion}

Venomous snakebite is an important, yet neglected public health problem in tropical countries, accounting for significant morbidity and mortality. In India, around 45,900 deaths are estimated to result annually from snakebites [1]. The commonly encountered venomous snakes in India include common cobra (Naja naja), common krait (Bungarus caeruleus), Russell's viper (Daboia russelli) and saw scaled viper (Echis carinatus), against which a locally prepared equine polyvalent ASV antitoxin is available. Neurological manifestations that follow envenomation by elapids (cobras and kraits) and less commonly Russell's viper include ptosis, external ophthalmoplegia,

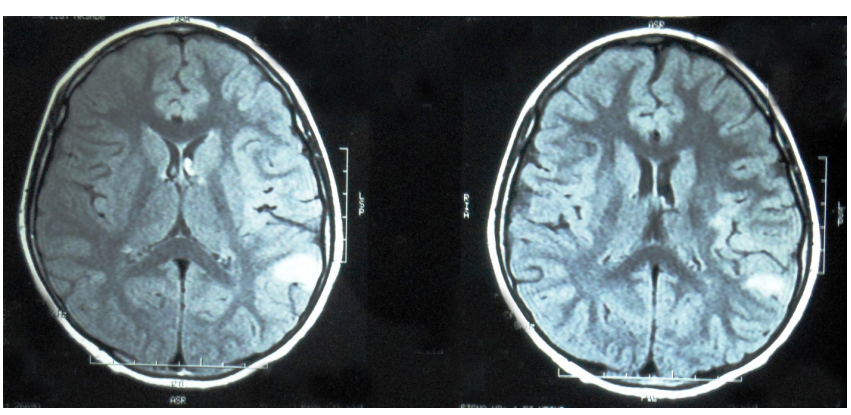

Fig.2: MRI brain revealed that there was left MCA acute infract with right parietal petechial hemorrhage.

paralysis of pharyngeal muscles, followed by respiratory and generalized muscle paralysis [2].

Neuromuscular paralysis occurs due to blockade of neuromuscular transmission. Cobra venom acts post-synaptically while krait venom acts pre-synaptically [1]. The binding to the presynaptic portion is irreversible, hence clinical recovery occurs slowly and only with the formation of a new neuromuscular junctions. The binding of toxin to the post-synaptic portion produces a competitive or non-competitive acetylcholine receptor blockade $[3,4]$. Although an anti-venom may induce a certain degree of reversal of the paralysis by post-synaptic neurotoxin, the clinical recovery is, however, very slow. Paralysis involves the proximal muscles first and then the distal muscles and recovery occurs in the reverse order [5].

In our present case it was common krait bite, as per history given by the patient's attendents and evident from progression of initial neurological symptoms. Initial neuro-paralytic symptoms resolved within 24 hours of bite with treatment, that indirectly supports the classical krait enovenomation pattern. But atypical to all this is the late sequalae, the acute infarct involving left MCA territory and petechial hemorrhage in parietal lobe. Central nervous system (CNS) hemorrhage within first few days of viper bite evolving into catastrophic consequences due to severe coagulopathy and as a part of DIC has been reported previously in 
various literature [6,7]. But vasculotoxic features developing in a patient of krait bite is quite rare and unique. Vasculotoxic feature in this patient is evident from local reaction, prolonged WBCT, PT, $\mathrm{CT}$, and abnormally elevated INR.

The etio-pathology of acute ischemic infarct is very hard to establish in this patient. The boy neither had any pre-existing source of embolization, nor any hyper-coagulability predisposing systemic condition or illness, as evident from the evaluation performed. Isolated CNS major arterial occlusion has not been reported as a part of DIC till date. Isolated bulbar encephalitis as a consequence of systemic envenomation was previously reported, but here the patient had no features of bulbar involvement. Vasculotoxic envenomation presenting with CNS hemorrhage is not very uncommon, but only major CNS artery occlusion following snake bite is quite rare. No features of encephalitis or meningoencephalitis were present in this patient. It could be a manifestation of vasculitis involving CNS vessels following systemic vasculotoxic envenomation. Though the snake venom was not isolated chemically, but from the clinical course of the illness it is quite evident that the patient had both neuroparalytic as well as vasculotoxicity features, which is quite rare (and occasionally evident in krait bites). Hence, it's a lesson from this case that vasculotoxic features can appear late in the course of the disease with krait bite which can lead not only to hemorrhage but also vasculitis. While treating such patients in future we have to study in details the manifestations and etiopathology behind these manifestations for better management of the patients.

\section{Conclusion}

Krait snake bite from circumferential evidence, a proper photographic evidence of the snake would have been better to sum up the conclusion which is unfortunately unavailable as most of instances. Never the less such bizarre central nervous system manifestations from two different etiology from single snake bite will enrich our existing knowledge in literature.

Contributors: MSH wrote the discussion and references, radiological confirmation of stroke and literature search. KG and SC managed the patient in secondary and tertiary care and wrote the case history. AB helped in literature search and treatment protocol. KG will act as guarantor of study. All authors approved the final version of this manuscript.

Funding: None; Competing interests: None stated.

\section{References}

1. Mohapatra B, Warrell DA, Suraweera W, Bhatia P, Dhingra N, Jotkar RM, et al. Snakebite mortality in India: a nationally representative mortality survey. PLoS Negl Trop Dis. 2011; 5:e1018.

2. Warrell DA. Snake bite. Lancet. 2010;375:77-88.

3. Abe T, Limbrick AR, Miledi R. Acute muscle denervation induced by b-bungarotoxin. Proc R Soc Lond B Biol Sci. 1976;194:545-553.

4. Awad SS, Lightowlers RR, Young C, ChrzanowskaLightowlers ZMA, Lømo T, Slater CR. Sodium channel mRNAs at the neuromuscular junction: distinct patterns of accumulation and effects of muscle activity. J Neurosci. 2001;21:8456-8463.

5. Ahmari SE, Buchanan J, Smith SJ. Assembly of presynaptic active zones from cytoplasmic transport packets. Nat Neurosci. 2000;3:445-451.

6. Yap $\mathrm{CH}$, Ihle BU. Coagulopathy after snake envenomation. Neurology. 2003;61:1788

7. Boyer LV, Seifert SA, Clark RF, McNally JT, Williams SR, Nordt SP, et al. Recurrent and persistent coagulopathy following pit viper evenomation. Arch Intern Med. 1999;159:706-710. 